\title{
NOTES ON JAN MARIA KISYŃSKI'S LIFE AND SCIENTIFIC WORK
}

A. Bobrowski, Lublin University of Technology, Lublin, Poland, a.bobrowski@pollub.pl

\section{The Beginnings}

Jan Maria Kisyński is a student of Adam Bielecki, whose PhD advisor was Witold Wilkosz, a close Cracow friend of Stefan Banach and Otto Nikodym. In early 1941, 5 years after Bielecki's PhD dissertation defense, Wilkosz who, though soon released after the infamous German "Sonderaktion Krakau", in which Polish Cracow elite professors were deceitfully captured and imprisoned in concentration camps, never recovered from serious health condition, died broken and impoverished. After the Second World War, Bielecki moved to Lublin to become one of the leaders of the newly formed Maria CurieSklodowska University there. Jan Kisyński was one of his first (and best) students.

As the latter reminisces, in Lublin he

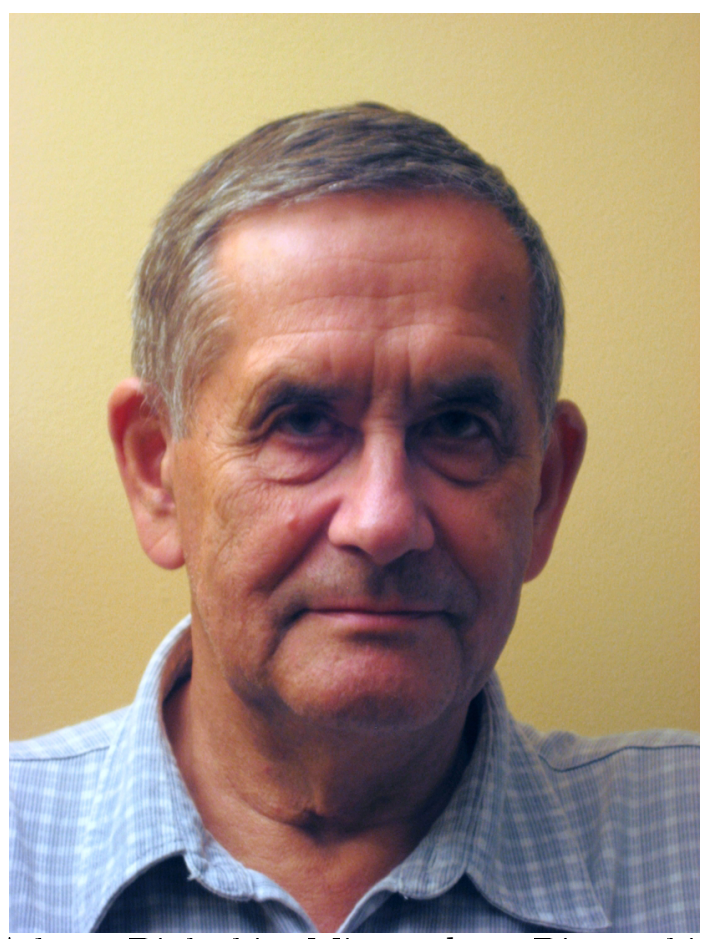
was taught by first-class analysts, including Adam Bielecki, Mieczysław Biernacki, Krzysztof Tatarkiewicz, Jan Krzyż, and Konstanty Radziszewski. All of them had impressively broad, comprehensive view not only on mathematics, but also on science and humanities in general. The atmosphere created by such great personalities undoubtedly helped to free up and develop a potential hidden in Kisyński's young mind. He always remained under a great spell of Bielecki's intellectual vigor. The latter cared a lot about his student's education, and often checked on him whether he studied sufficiently many academically profitable books. In particular, he urged him to read [59], and in fact gave him a copy of this monograph.

Before commencing the description of Jan Kisyński's scientific achievements, let me make a personal comment, which agrees well with his views. In fact, to all those who work in mathematics it should be amply clear that obtaining a meaningful, seminal result requires time: at least a few years, sometimes 10 years. Thus, it is impossible to write more than, say 20, important articles. This point of view is not so popular today, in the times of the publish or perish philosophy pressure, but I seem to meet more and 
more mathematicians who adhere to this view, especially those who are able to look at their achievements from a perspective. Hence, from the over-50-articles-long list of JK's publications I have chosen 9 which seem to be of greatest value. They are

$$
[38,40,41,44,46,49-51,53] \text {. }
$$

I will try to describe all of them in short, one by one, skimming over some other papers.

Kisyński's first series of articles, written under great influence of his master, Adam Bielecki, was devoted to quasilinear hyperbolic equations of two variables; but at the same time he also published on other subjects (see e.g., [34-37]).

A turning point in his scientific career comes in the late 1950s, when a renown functional analyst, Włodzimierz Mlak comes to Lublin to speak about new subject: differential equations in Banach spaces. Kisyński is intrigued, studies the first edition of Hille's book on functional analysis and semigroups of operators [29] (and, a little bit later Lions's [57]), and soon makes an entrance into the semigroup society with the influential article [38] on solvability of temporarily inhomogeneous Cauchy problems (see also the related [39]). The argument of [38] is repeated verbatim in the second edition of Yosida's fundamental treatise [66, Section XIV.4], and the article is cited in all subsequent editions of this monograph, and in the equally well-known monographs [56, Section II.2 and II.3] and [63]. By the way, the same article is the basis for Kisyński's habilitation; by that time JK has moved to Warsaw and is affiliated with the university there and with the Polish Academy of Sciences.

There is an anecdote related to Lions's book [57] mentioned above, which by the way was recommended to Jan Kisyński by Krzysztof Maurin (see below). As JK recalls, on one of his skiing trips to Tatra Mountains he took the book along. One day the weather was rather bad, and he had to stay in a mountain shelter in Hala Gąsienicowa. He was busy studying the book in a crowd of fellow tourists. Suddenly, somebody approached him and asked "Have they caught him yet?". As it transpired, it was Andrzej Skoczylas, a quite well-known alpinist, who apparently could not believe that one may read avidly anything else than crime stories.

A second stunning achievement soon follows: in 1967 his seminal article [40] with a short, elegant and surprising proof of Trotter-Kato's theorem on convergence of sequences of semigroups of operators is published. By considering a space of convergent, vector-valued sequences, Kisyński reduces the question of convergence to the question of generation of a semigroup in this space and thus is able to deduce the Trotter-Kato theorem from the Hille-Yosida theorem in one impressive move. This approach not only soon makes its way into fundamental monographs on semigroups of operators (see e.g., [26, Section 1.7] or [19, Section III.4]) but also allows a bird's-eye view of the question of convergence, and a deeper understanding of phenomena accompanying convergence if the semigroups converge merely on a subspace of the underlying Banach space (see [7, Chapter 14]).

To all those who know Jan Kisyński only from his work in semigroup theory, his third influential paper may come as a great surprise, but in a sense the paper characterizes the author better than his other works. JK has always viewed mathematics as a whole, and only "when forced" described himself as a functional analyst. During his time in Warsaw, thus, he was greatly influenced by Krzysztof Maurin (a great Polish analyst, mathematician, writer and philosopher - JK often commented on Maurin's long-lasting influence on him and on Polish mathematics as a whole) and Bogdan Bojarski, whose seminar Kisyński 
frequented. It was the former who made Kisyński interested in the problem of generation of tight measures: the resulting paper [41] appeared in 1968 and now forms an integral part of the canon of measure theory (see e.g., [18, Section VII.2] and [3]). A characteristic and a great asset of the paper is that in it the author does not follow a well-trodden path but forces his way through a rather unknown territory, using new and non-classical arguments: instead of commonly used outer measure, he consistently works with inner measure.

\section{The 1970s}

The beginning of 1970s, following the fundamental work of Miroslav Sova on operatorvalued cosine functions [64] (see also [60] apparently unknown to JK at the time), is marked by a beautiful series of Jan Kisyński's papers devoted to second-order abstract Cauchy problems in Banach spaces. In the first of these [42], he studies convergence of solutions of

$$
\epsilon u(t)+u^{\prime \prime}(t)=A u(t)
$$

to those

$$
u^{\prime \prime}(t)=A u(t)
$$

as $\epsilon \rightarrow 0$, where $A$ is assumed to generate a cosine family. The main result, obtained by an ingenious analysis of Laplace transforms and involving completely monotone functions, says that the distance between these solutions can be estimated by that between solutions of corresponding scalar equations (with $A$ replaced by a positive number). Years later in 1991, JK has made a more comprehensive analysis of the full second order equation

$$
a u(t)+b u^{\prime \prime}(t)+c u(t)=A u(t),
$$

but the result was published in Polish, and in a local journal in Lublin [50], so that it is not available to a broader international readership (see, however, pp. 134-149 of this special issue). The fact that $c$ in (2) is in general non-zero, is important in the theory of neuron transport, and the lack of care in dealing with initial and boundary layers involved in approximating solutions of (2) by those of (1) may lead to serious consequences, like these we know from the Chernobyl disaster.

By the way, Jan Kisyński never cared much to publish his results in "leading" journals. Thus, a number of interesting results were published in Prace Naukowe Politechniki Lubelskiej (Research Bulletin of Lublin University of Technology; he returned to Lublin in the mid 1980s). An anecdote says that one of those papers was to be sent to Wojciech Chojnacki for a review. Despite the fact that the latter resides in Australia, a bureaucrat decided to save on post stamps and sent the paper by regular mail. Thus, instead of by plain, the manuscript traveled comfortably by ship, due to the mercy of the sailors was not thrown overboard, and arrived safely in Adelaide only several months later.

In the next two 1970s papers [43,45], the question of representability of an operator cosine function in the form

$$
\operatorname{Cos}(t)=\frac{1}{2}(G(t)+G(-t)), \quad t \in \mathbb{R},
$$

where $G$ is a group of operators is studied in depth. Independently, the same problem was approached (from a slightly different angle) by H.O. Fattorini [21,22]. This apparently 
esoteric subject aroused surprisingly large interest (for example of the great Czesław RyllNardzewski, who contributed a variant of the proof of one of the theorems) and was later greatly developed by JK's student, Wojciech Chojnacki (see e.g., [10] and the references given there); one of the best-known recent results in this field, though, seems to belong to Markus Haase [27]. The papers [43,45] are of course referred to repeatedly in Fattorini's monograph [23] but are relatively less known to the Western mathematical community than the theorem we will discuss next.

The main idea of the paper [44] is pretty straightforward: a second order Cauchy's problem should be reducible to a first order system of equations of two unknowns (the second unknown is the derivative). But, as the old saying goes, the devil is in the detail, and the previous, less successful attempts (e.g., by the already cited Fattorini [21,22]) show that the matter is not so trivial as it may seem. A corollary to Kisyński's main theorem may be phrased as follows. Let $\left\{\operatorname{Cos}_{A}(t), t \in \mathbb{R}\right\}$ be a strongly continuous cosine family in a Banach space $\mathbb{X}$, generated by an operator $A$. Then, there is a Banach space $\mathbb{X}_{\text {Kis }}$ which is uniquely determined up to the definition of the underlying norm, such that

(i) $\mathcal{D}(A) \subset \mathbb{X}_{\mathrm{Kis}}$,

(ii) $\mathbb{X}_{\text {Kis }}$ is continuously embedded in $\mathbb{X}: \mathbb{X}_{\text {Kis }} \hookrightarrow \mathbb{X}$,

(iii) for $x \in \mathbb{X}_{\mathrm{Kis}}, t \mapsto \operatorname{Cos}_{A}(t) x$ is continuously differentiable,

(iv) the operators

$$
\left(\begin{array}{ll}
\left(\operatorname{Cos}_{A}(t)\right)_{\mid \mathbb{X}_{\mathrm{Kis}}} & \int_{0}^{t} \operatorname{Cos}_{A}(s) \mathrm{d} s \\
\left(\frac{\mathrm{d} \operatorname{Cos}_{A}(t)}{\mathrm{d} t}\right)_{\mid \mathbb{X}_{\mathrm{Kis}}} & \operatorname{Cos}_{A}(t)
\end{array}\right), \quad t \in \mathbb{R}
$$

form a one-parameter group in $\mathbb{X}_{\text {Kis }} \times \mathbb{X}$.

From (3) it is clear that the generator of the latter group is $\mathcal{A}(y, x)=(x, A y)$ with domain $\mathcal{D}(\mathcal{A})$ composed of $(y, x)$ with $y \in \mathcal{D}(A), x \in \mathbb{X}_{\text {Kis }}$. It is also manifest that the corollary is a deeper description of what a cosine operator function is than that given by Sova, da Prato and Giusti. For one thing, it provides an information of odd-order derivatives of $t \mapsto \operatorname{Cos}_{A}(t)$ which Sova-daPrato-Giusti theorem by nature cannot do.

The force of this result lies also in the fact, which we want to stress here again, that $\mathbb{X}_{\text {Kis }}$ is uniquely determined (but not if conditions (i)-(ii) are omitted; see [58].) Nowadays $\mathbb{X}_{\mathrm{Kis}}$ is often referred to as the Kisyński space, while $\mathbb{X}_{\mathrm{Kis}} \times \mathbb{X}$ is called the phase space, and it has become amply clear that these notions play a central role in proving generation theorems for cosine families (see e.g. [1, Section 3.14], [4] or [11]). In particular, attempts at proving theorems via analysis in the Cartesian product $\mathbb{X} \times \mathbb{X}$ are not as fruitful.

The next result we want to discuss is perhaps less known but ever so beautiful. In fact it is also somewhat unusual. To recall, since the time of publication of the pioneering paper by Kolmogorov [54], it has been well known that there is a close connection between stochastic processes and partial differential equations of second order. Partial differential equations of second order form three distinct classes: the elliptic, the parabolic and the hyperbolic equations. One of the reasons for such a classification is the fact that properties of PDEs differ greatly depending on which class they belong to. Now, the second order PDEs that are known to be related to stochastic processes are of elliptic or parabolic type (see e.g. [15-17,24,65]). The process that we are going to describe now, however, is related to a hyperbolic PDE known as the telegraph equation. A probabilistic formula for the solutions to this equation was introduced by Samuel Goldstein [25] and Mark 
Kac [33]. We note that the discovery of Kac, followed by papers by R. J. Griego and R. Hersh, marked the beginning of interest in so-called random evolutions (see e.g. [61] where also an abundant bibliography is given). Yet later, this class grew into piece-wise deterministic processes of M.H.A. Davies [13,14], which are indispensable in modeling biological phenomena [62].

Let me describe the result obtained by Kac in more detail. Let $a>0$ and $v$ be two real numbers. The equation

$$
\frac{\partial^{2} y(t, \tau)}{\partial t^{2}}+2 a \frac{\partial y(t, \tau)}{\partial t}=v^{2} \frac{\partial^{2} y(t, \tau)}{\partial \tau^{2}}
$$

is called the telegraph equation. From the theory of PDEs it is known that it has exactly one solution if we require additionally that

$$
y(0, \tau)=y(\tau), \quad \text { and } \quad \frac{\partial y(0, \tau)}{\partial t}=0,
$$

where $y$ is a sufficiently regular function. M. Kac has shown that the solution is given by:

$$
y(t, \tau)=\frac{1}{2} \mathbb{E}[y(\tau+v \xi(t))+y(\tau-v \xi(t))], \quad t \geq 0, \tau \in \mathbb{R}
$$

where

$$
\xi(t)=\xi_{a}(t)=\int_{0}^{t}(-1)^{N(u)} \mathrm{d} u, \quad t \geq 0,
$$

$\{N(t), t \geq 0\}$ is a Poisson process with parameter $a$, and $\mathbb{E}$ denotes expected value. This formula generalizes the solution to the wave equation

$$
\frac{\partial^{2} y(t, \tau)}{\partial t^{2}}=v^{2} \frac{\partial^{2} y(t, \tau)}{\partial \tau^{2}}
$$

which is well-known to be

$$
y(t, \tau)=\frac{1}{2}[y(\tau+v t)+y(\tau-v t)], \quad t \geq 0, \tau \in \mathbb{R} .
$$

For, the only difference between the telegraph equation and the wave equation is the second term on the left-hand side of (4); and if $a=0$ the telegraph equation becomes the wave equation. In such a case, however, the Poisson process degenerates to a family of random variables that are all equal to 0 , and thus $\int_{0}^{t}(-1)^{N(u)} \mathrm{d} u=t$ (a.s.), so that (5) reduces to (7).

Of course, formula (7) involves (as it must) a cosine function

$$
C(t) f(\tau)=\frac{1}{2}(f(\tau+v t)+f(\tau-v t)), \quad t \in \mathbb{R}, \tau \in \mathbb{R},
$$

of operators in, say, the space $B U C(\mathbb{R})$ of bounded, uniformly continuous functions $f$ on $\mathbb{R}$. In terms of this family, (5) takes the form:

$$
y(t, \tau)=\mathbb{E} C(\xi(t)) y(\tau) .
$$


In [46], generalizing these results, J. Kisyński shows that if $A$ is the generator of a strongly continuous cosine family $\{C(t), t \in \mathbb{R}\}$ in a Banach space $\mathbb{X}$, then the solution of the abstract Cauchy problem

$$
u(t)+2 a u^{\prime \prime}(t)=A u(t), \quad t \geq 0
$$

with initial conditions $u(0)=u_{0}, u^{\prime \prime}(0)=u_{1}$ is

$$
u(t)=\mathbb{E} C(\xi(t)) u_{0}+\mathbb{E} \int_{0}^{\xi(t)} C(s) u_{1} \mathrm{~d} s, \quad t \geq 0 .
$$

A stunningly simple and elegant formula!

But the value of the paper in question goes far beyond this. For the key step in the argument presented in [46] involved an analysis of the process

$$
\left(\xi(t),(-1)^{N(t)}\right)
$$

which was shown to have independent increments in the non-commutative group $\mathbb{R} \times$ $\{-1,1\}$ with the following multiplication rule:

$$
(\tau, k) \circ(\varsigma, l)=(\tau l+\varsigma, k l) .
$$

The importance of this example was soon recognized by the specialists of the then booming theory of Lévy processes on locally compact groups (see e.g., the introduction to the Russian translation of Heyer's monograph [28] published by MIR in 1981), the result made its way into treatises like [20] and is nowadays very well-known (see e.g. [32] or [55]). It is also perhaps of interest to note that the result of [42] discussed earlier implies that properly rescaled telegraph process (6) converges in distribution to the Brownian motion (see also [61] for this subject).

In 1976, JK's Trieste lecture notes on semigroups of operators were published [47]. Although over 40 years have passed since that time, the notes are still a valuable source of information on the subject and are often referred to by students and specialists. Notably, they contain a number of non-standard examples which cannot be found elsewhere. The presentation is also quite unique: even well-known results are often proved in surprising, elegant ways. By that time JK was appointed a state professor.

The 1970s were crowned with the important article [49], not related to second order Cauchy problems, but devoted to semigroups generated by differential operators on Lie groups, a subject quite natural for the author, since the the group described above in the presentation of the telegraph equation, bearing now his name, is of course an example of a Lie group. It is worth noting that the article was published in the Journal of Functional Analysis and communicated by Lars Gårding himself - an honor not many had. There is an anecdote related to this article worth recording here. Włodzimierz Mlak commented once to JK that he likes to read his articles for clarity and style, but dislikes this particular one. JK answered that it is Gårding who is to be blamed for that - he accepted the article on the condition that it will not be longer than 15 pages or so; JK duly shortened it to 11 pages (and made it less readable).

Another article worth noting here is [48]. This is a trace of JK's long-lasting, nearly 20 years long, interest in and diligent work on the quasi-classical approximation in quantum physics. 


\section{More Recent Work}

Let me complete this survey with basic information on two most influential papers of Jan Kisyński published in the last twenty years. The first of these is [51]. To explain its main idea let us consider a not necessarily densely defined linear operator $A$ in a Banach space $\mathbb{X}$ such that all $\lambda>0$ belong to the resolvent set of $A$ and

$$
M:=\sup _{\lambda>0, n \geq 1}\left\|\lambda^{n}(\lambda-A)^{-n}\right\|<\infty
$$

such operators are nowadays called Hille-Yosida operators. It is well-known that a HilleYosida operator generates a strongly continuous semigroup on the closure of its domain, called the regularity space:

$$
\mathbb{X}^{\prime}=\operatorname{cl} \mathcal{D}(A)
$$

outside of $\mathbb{X}^{\prime}$ the semigroup is in general undefined but there exists an object called a Lipschitz continuous integrated semigroup [1].

Kisyński proves that condition (8) is equivalent to the existence of a representation

$$
H: L^{1}\left(\mathbb{R}^{+}\right) \rightarrow \mathcal{L}(\mathbb{X})
$$

of the convolution algebra $L^{1}\left(\mathbb{R}^{+}\right)$of absolutely integrable functions on $\mathbb{R}^{+}$, by bounded linear operators in $\mathbb{X}$, such that $\|H\|=M$ and

$$
H\left(e_{\lambda}\right)=(\lambda-A)^{-1}, \quad \lambda>0,
$$

where $e_{\lambda}$ is given by $e_{\lambda}(\tau)=\mathrm{e}^{-\lambda \tau}, \tau \geq 0$ (see [30] for a related result). Also, he characterized the regularity space and the semigroup defined there as follows: $x$ is a member of $\mathbb{X}^{\prime}$ iff there are $\phi \in L^{1}\left(\mathbb{R}^{+}\right)$and $y \in \mathbb{X}$ such that $x=H(\phi) y$ (in other words, $\mathbb{X}^{\prime}$ is the range of the representation $H$ ) and the semigroup generated by $A$ is given by

$$
T(t) x=H\left(\phi_{t}\right) y, \quad t \geq 0,
$$

where $\phi_{t}$ is the translation of $\phi \in L^{1}\left(\mathbb{R}^{+}\right)$to the right.

Besides indisputable beauty and elegance of this algebraic version of the Hille-Yosida theorem, the importance of this result lies in the fact that it provides an intriguing link between the theory of semigroups of operators and the celebrated factorization theorem of P.J. Cohen [12] (see also [31, Section 32]). Namely, the characterization of the regularity space given above is possible since, by the Cohen theorem, the range of $H$ is a closed set, $L^{1}\left(\mathbb{R}^{+}\right)$having a bounded approximate identity (see $[8,9,52]$ for more information). JK often stresses that originally, being unaware of Cohen's result, he characterized the regularity space as the closure of the range of $H$ - it was W. Chojnacki, who turned his attention to the factorization theorem. He also comments (to my delight) that his work on the algebraic version was influenced by my papers $[5,6]$. To use his own words: "It is a measure of my success that I had co-worked with such mathematicians; they are of too high a class for me to treat them simply as my students."

It should be stressed that the result obtained in [51] provided a new, and unexpected insight into Wolfgang Arendt's theory of integrated semigroups [1] and lead naturally to the analysis of distribution semigroups. The latter objects were studied in the seminal paper [53] in the Journal of Functional Analysis, communicated by P. Malliavin. As 
commented by Valentin Keyantuo in a very detailed Mathematical Review article, in [53] Kisyński develops "a theory of distribution and ultradistribution semigroups based on the Fourier transform and a careful analysis of pseudo-resolvents". Such semigroups are strongly related to local integrated semigroups (see e.g. [2] which JK values very highly) which "give a way to study Cauchy problems that may not be well posed in the classical sense". "The results include the case in which the distribution semigroups may be degenerate, this being used to study implicit Cauchy problems". V. Keyantuo concludes by saying: "The approach is elegant, very general and provides a unified framework for various previous results. Concrete problems (...) are dealt with successfully". Needless to say, [53] was followed by a long series of other articles devoted to related subjects.

\section{References}

1. Arendt W., Batty J.K., Hieber M., Neubrander F. Vector-Valued Laplace Transforms and Cauchy Problems. Basel, Birkhäuser, 2001. DOI: 10.1007/978-3-0348-5075-9

2. Arendt W., El-Mennaoui O., Kéyantuo V. Local Integrated Semigroups: Evolution with Jumps of Regularity. Journal of Mathematical Analysis and Applications, 1994, vol. 186, no. 2, pp. 572-595. DOI: 10.1006/jmaa.1994.1318

3. Berg C., Christensen J.P.R., Ressel P. Harmonic Analysis on Semigroups. Theory of Positive Definite and Related Functions. N.Y., Springer, 1984. DOI: 10.1007/978-1-4612-1128-0

4. Bátkai A., Engel K.-J. Abstract Wave Equations with Generalized Wentzell Boundary Conditions. Journal of Differential Equations, 2004, vol. 207, no. 1, pp. 1-20. DOI: $10.1016 /$ j.jde.2003.12.005

5. Bobrowski A. Integrated Semigroups and the Trotter-Kato Theorem. Bulletin of the Polish Academy of Sciences Mathematics, 1994, vol. 41, pp. 297-304.

6. Bobrowski A. On the Yosida Approximation and the Widder-Arendt Theorem. Studia Mathematica, 1997, vol. 124, no. 3, pp. 281-290. DOI: 10.4064/sm-124-3-281-290

7. Bobrowski A. Convergence of One-Parameter Operator Semigroups. In Models of Mathematical Biology and Elsewhere. Cambridge, Cambridge University Press, 2016. DOI: $10.1017 / \mathrm{CBO} 9781316480663$

8. Chojnacki W. On the Equivalence of a Theorem of Kisyński and the Hille-Yosida Generation Theorem. Proceedings of the American Mathematical Society, 1998, vol. 126, no. 2, pp. 491-497. DOI: 10.1090/S0002-9939-98-04048-9

9. Chojnacki W. Multiplier Algebras, Banach Bundles, and One-Parameter Semigroups. Annali della Scuola Normale Superiore di Pisa - Classe di Scienze, 1999, vol. 28, no. 2, pp. 287-322.

10. Chojnacki W. On Operator-Valued Cosine Sequences on UMD Spaces. Studia Mathematica, 2010, vol. 199, no. 3, pp. 267-278. DOI: 10.4064/sm199-3-4

11. Chill R., Keyantuo V., Warma M. Generation of Cosine Families on $L^{p}(0,1)$ by Elliptic Operators with Robin Boundary Conditions. Functional Analysis and Evolution Equations. The Günter Lumer Volume, Basel, Birkhäuser, 2007, pp. 113-130. DOI: 10.1007/978-3-76437794-6_7

12. Cohen P.J. Factorization in Group Algebras. Duke Mathematical Journal, 1959, vol. 26, no. 2, pp. 199-205. DOI: 10.1215/S0012-7094-59-02620-1

13. Davis M.H.A. Lectures on Stochastic Control and Nonlinear Filtering. N.Y., Springer, 1984.

14. Davis M.H.A. Markov Processes and Optimization. London, Chapman and Hall, 1993.

15. Durrett R. Stochastic Calculus. A Practical Introduction. Boca Raton, CRC Press, 1996. 
16. Dynkin E.B. Markov Processes. Vols. I, II. Berlin, Göttingen, Heidelberg, Springer 1965. DOI: $10.1007 / 978-3-662-00031-1$

17. Ethier S.N., Kurtz T.G. Markov Processes. Characterization and Convergence. N.Y., Wiley, 1986. DOI: $10.1002 / 9780470316658$

18. Elstrodt J. Maß- und Integrationstheorie. Berlin, Heidelberg, Springer, 2005. (in German) DOI: $10.1007 / \mathrm{b} 137969$

19. Engel K.-J., Nagel R. One-Parameter Semigroups for Linear Evolution Equations. N.Y., Springer, 2000.

20. Ermakov S.M., Nekrutkin V.V., Sipin A.S. Random Processes for Classical Equations of Mathematical Physics. Dordrecht, Kluwer Academic Publishers Group, 1989. DOI: $10.1007 / 978-94-009-2243-3$

21. Fattorini H.O. Ordinary Differential Equations in Linear Topological Spaces. I. Journal of Differential Equations, 1969, vol. 5, pp. 72-105. DOI: 10.1016/0022-0396(69)90105-3

22. Fattorini H.O. Ordinary Differential Equations in Linear Topological Spaces. II. Journal of Differential Equations, 1969, vol. 6, pp. 50-70. DOI: 10.1016/0022-0396(69)90117-X

23. Fattorini H.O. Second Order Linear Differential Equations in Banach Spaces. Amsterdam, North-Holland, 1985.

24. Freidlin M. Functional Integration and Partial Differential Equations. Princeton, Princeton University Press, 1985.

25. Goldstein S. On Diffusion by Discontinuous Movements, and on the Telegraph Equation. The Quarterly Journal of Mechanics and Applied Mathematics, 1951, no. 4, pp. 129-156.

26. Goldstein J.A. Semigroups of Linear Operators and Applications. N.Y., Oxford University Press, 1985.

27. Haase M. The Group Reduction for Bounded Cosine Functions on UMD Spaces. Mathematische Zeitschrift, 2009, vol. 262, no. 2, pp. 281-299. DOI: 10.1007/s00209-008$0373-\mathrm{y}$

28. Heyer H. Probability Measures on Locally Compact Groups. Berlin, Heidelberg, Springer, 1977. DOI: $10.1007 / 978-3-642-66706-0$

29. Hille E. Functional Analysis and Semi-Groups. N.Y., American Mathematical Society, 1948.

30. Hennig B., Neubrander F. On Representations, Inversions and Approximations of Laplace Transform in Banach Spaces. Applicable Analysis, 1993, vol. 49, pp. 151-170.

31. Hewitt E., Ross K.A. Abstract Harmonic Analysis. Vol. II: Structure and Analysis for Compact Groups. Analysis on Locally Compact Abelian Groups. Berlin, Heidelberg, Springer, 1970. DOI: $10.1007 / 978-3-662-26755-4$

32. Janssen A., Siebert E. Convolution Semigroups and Generalized Telegraph Equations. Mathematische Zeitschrift, 1981, vol. 177, no. 4, pp. 519-532. DOI: 10.1007/BF01219084

33. Kac M. Some Stochastic Problems in Physics and Mechanics. Colloq. Lect. 2, Magnolia Petrolum, 1956.

34. Kisyński J. Sur la méthode des approximations successives pour un systéme de $n$ équations à $n$ inconnues. Bull. Acad. Polon. Sci. Sér. Sci. Math. Astr. Phys., 1958, vol. 6, pp. 683-687. (in French)

35. Kisyński J. Sur les equations differentielles dans les espaces de Banach. Bull. Acad. Polon. Sci. Sér. Sci. Math. Astr. Phys., 1959, vol. 7, pp. 381-385. (in French)

36. Kisyński J. Convergence du type $\mathcal{L}$. Colloquium Mathematicum, 1959/1960, vol. 7, pp. 205-211. (in French) DOI: 10.4064/cm-7-2-205-211 
37. Kisyński J. Sur les familles compactes de fonctions mesurables. Colloquium Mathematicum, 1959/1960, vol. 7, pp. 221-235. (in French) DOI: 10.4064/cm-7-2-221-235

38. Kisyński J. Sur les opérateurs de Green des problémes de Cauchy abstraits. Studia Mathematica, 1963/1964, vol. 23, pp. 285-328. (in French) DOI: 10.4064/sm-23-3-285-328

39. Kisyński J. On Temporally Inhomogeneous Equations of Evolution in Banach Spaces. Bull. Acad. Polon. Sci. Sér. Sci. Math. Astr. Phys., 1966, vol. 14, pp. 623-626.

40. Kisyński J. A Proof of the Trotter-Kato Theorem on Approximation of Semigroups. Colloquium Mathematicum, 1967, vol. 18, pp. 181-184. DOI: 10.4064/cm-18-1-181-184

41. Kisyński J. On the Generation of Tight Measures. Studia Mathematica, 1968, vol. 30, pp. 141-151. DOI: $10.4064 / \mathrm{sm}-30-2-141-151$

42. Kisyński J. On Second Order Cauchy's Problem in a Banach Space. Bull. Acad. Polon. Sci. Sér. Sci. Math. Astr. Phys., 1970, vol. 18, no. 7, pp. 371-374.

43. Kisyński J. On Operator-Valued Solutions of d'Alembert's Functional Equation. I. Colloquium Mathematicum, 1971, vol. 23, pp. 107-114. DOI: 10.4064/cm-23-1-107-114

44. Kisyński J. On Cosine Operator Functions and One-Parameter Groups of Operators. Studia Mathematica, 1972, vol. 44, pp. 93-105. DOI: 10.4064/sm-44-1-93-105

45. Kisyński J. On Operator-Valued Solutions of d'Alembert's Functional Equation. II. Studia Mathematica, 1972, vol. 42, pp. 43-66.

46. Kisyński J. On M. Kac's Probabilistic Formula for the Solutions of the Telegraphist's Equation. Annales Polonici Mathematici, 1974, vol. 29, pp. 259-272. DOI: 10.4064/ap-29$3-259-272$

47. Kisyński J. Semi-Groups of Operators and Some of Their Applications to Partial Differential Equations. Control Theory and Topics in Functional Analysis (Internat. Sem., Internat. Centre Theoret. Phys., Trieste, 1974), Vienna, International Atomic Energy Agency, 1976, vol. 3, pp. 305-405.

48. Kisyński J. The Argument of a Lagrange Subspace and the Maslov Index. Institute of Mathematics, Polish Academy of Sciences, Preprint 202, 1979.

49. Kisyński J. On Semigroups Generated by Differential Operators on Lie Groups. Journal of Functional Analysis, 1979, vol. 31, no. 2, pp. 234-244. DOI: 10.1016/0022-1236(79)90064-8

50. Kisyński J. O równaniu telegrafistów z małym parametrem [On the Telegraph Equation with a Small Parameter]. Prace Naukowe Politechniki Lubelskiej 212, Matematyka [Research Bulletin of Lublin University of Technology], 1991, vol. 12, pp. 14-27. (in Polish)

51. Kisyński J. The Widder Spaces, Representations of the Convolution Algebra $L^{1}\left(\mathbb{R}^{+}\right)$and One Parameter Semigroups of Operators. Institute of Mathematics, Polish Academy of Sciences, 1998, Preprint 588, pp. 1-36.

52. Kisyński J. On Cohen's Proof of the Factorization Theorem. Annales Polonici Mathematici, 2000, vol. 75, no. 2, pp. 177-192. DOI: 10.4064/ap-75-2-177-192

53. Kisyński J. On Fourier Transforms of Distribution Semigroups. Journal of Functional Analysis, 2007, vol. 242, pp. 400-441. DOI: 10.1016/j.jfa.2006.07.003

54. Kolmogorov A.N. Über die analytischen Methoden in der Wahrscheinlichkeitsrechnung. Mathematische Annalen, 1931, vol. 104, no. 1, pp. 415-458. (in German) DOI: $10.1007 / \mathrm{BF} 01457949$

55. Kolesnik A.D. The Explicit Probability Distribution of the Sum of Two Telegraph Processes. Stochastics and Dynamics, 2015, vol. 15, no. 2, article ID: 1550013, 32 p. 
56. Krein S.G. Linear Differential Equations in Banach Space. Providence, American Mathematical Society, 1971.

57. Lions J.-L. Équations différentielles opérationnelles et problémes aux limites. Die Grundlehren der mathematischen Wissenschaften, Berlin, Góttingen, Heidelberg, Springer, 1961. (in French)

58. Lax P.D., Phillips R.S. Scattering Theory. N.Y., Academic Press, 1967.

59. Natanson I.P. Teoriya funkciy veshchestvennoy peremennoy [Theory of Functions of Real Variable]. Moscow, Gosudarstvennoe izdatel'stvo tekhnichesko-teoreticheskoy literatury, 1957. (in Russian)

60. Da Prato G., Giusti F. Una carracterizzazione dei generatori di funzioni coseno astratte. Bollettino dell'Unione Matematica Italiana, 1967, vol. 22, pp. 357-362. (in Italian)

61. Pinsky M.A. Lectures on Random Evolutions. Singapore, World Scientific, 1991. DOI: $10.1142 / 1328$

62. Rudnicki R., Tyran-Kamińska M. Piecewise Deterministic Processes in Biological Models. Springer International Publishing, 2017. DOI: 10.1007/978-3-319-61295-9

63. Simon B. Quantum Mechanics for Hamiltonians Defined as Quadratic Forms. Princeton, Princeton University Press, 1971.

64. Sova M. Cosine Operator Functions. Rozprawy Matematyczne, 1966, vol. 49, pp. 1-47.

65. Wentzell A.D. A Course in the Theory of Stochastic Processes. N.Y., McGraw-Hill International Book, 1981.

66. Yosida K. Functional Analysis. Berlin, Springer, 1965.

Received May 27, 2018 